\title{
Rapid Bound State Formation of Dark Matter in the Early Universe
}

\author{
Tobias Binder $\oplus^{1,{ }^{*}}$ Kyohei Mukaida, ${ }^{2, \dagger}$ and Kalliopi Petraki $\oplus^{3,4,+}$ \\ ${ }^{1}$ Kavli IPMU (WPI), UTIAS, The University of Tokyo, Kashiwa, Chiba 277-8583, Japan \\ ${ }^{2}$ Deutsches Elektronen-Synchrotron (DESY), Notkestraße 85, Hamburg D-22607, Germany \\ ${ }^{3}$ Sorbonne Université, CNRS, Laboratoire de Physique Théorique et Hautes Energies, LPTHE, F-75252 Paris, France \\ ${ }^{4}$ Nikhef, Science Park 105, 1098 XG Amsterdam, The Netherlands
}

(Received 6 December 2019; revised manuscript received 19 February 2020; accepted 18 March 2020; published 21 April 2020)

\begin{abstract}
The formation and decay of dark matter (DM) bound states deplete the thermal relic density during the chemical decoupling process, allowing for larger DM masses. While so far the bound state formation (BSF) has been described via the emission of an on-shell mediator, we point out that this particular process does not have to be the dominant one in general. If the mediator is coupled in a direct way to any relativistic species present in the early Universe, we demonstrate that BSF can much more efficiently occur through particle scattering. Consequently, DM can be heavier than previously expected.
\end{abstract}

DOI: 10.1103/PhysRevLett.124.161102

Introduction.-One of the leading dark matter (DM) hypotheses is that it consists of weakly interacting massive particles (WIMPs) [1-3], which can explain the observed DM abundance [4] in a natural way through the thermal production mechanism. While strong upper bounds on the coupling strength of the WIMP to standard model (SM) particles, derived from direct, indirect and collider searches, rule out many electroweak mass-scale realizations in the thermal production scenario, the $\mathrm{TeV}$ mass region and above still remains an attractive and much less constrained possibility.

The heavier WIMPs are, the more important it is to include quantum mechanical effects induced by long-range interactions in the thermal decoupling description for predicting the relic abundance accurately. In a seminal work [5], it was pointed out that already around a $3 \mathrm{TeV}$ mass, even the heaviest massive gauge bosons of the SM effectively mediate attractive long-range forces between a WIMP pair, leading to an enhanced annihilation cross section during the freeze-out process. This so-called Sommerfeld enhancement (SE) [6], or Sakharov enhancement $[7,8]$, lowers the predicted thermal relic abundance compared to a tree-level computation, which in turn allows for larger masses of the annihilating DM particles to compensate for the effect (see, e.g., Refs. [9-17], and Refs. [18-24] for formal aspects).

Further quantum mechanical effects caused by attractive long-range interactions are bound state solutions in the

Published by the American Physical Society under the terms of the Creative Commons Attribution 4.0 International license. Further distribution of this work must maintain attribution to the author(s) and the published article's title, journal citation, and DOI. Funded by SCOAP ${ }^{3}$. two-particle spectrum of WIMPs [25-27]. The formation of these bound states and their subsequent decay into SM particles additionally depletes the relic density, allowing for even heavier masses [28]. For every SM force carrier, possible DM scenarios have been found in the literature where the inclusion of the SE and bound state formation (BSF) is relevant. Famous examples in supersymmetric extensions of the SM are cases where DM coannihilates with closely mass degenerate partners, which are either electroweakly or color charged [29-39]. In addition to the photon, gluon, $Z$ or $W^{ \pm}$boson induced bound states in mentioned cases, also the Higgs boson [40-42] can attractively contribute to confining DM into metastable bound states, even for otherwise repulsive color octet states [42]. Furthermore, these quantum mechanical phenomena certainly play a role in bottom-up motivated scenarios $[43,44]$, e.g., self-interacting DM and other models with light mediators [45-61].

So far, the DM BSF in the early Universe has been described via the emission of an on-shell mediator. While this resembles the situation of SM neutral hydrogen recombination in the matter-dominated epoch, an interesting question is by how much scattering events with primordial plasma constituents in the radiation dominated epoch could additionally stimulate the conversion process through virtual mediator exchanges.

In the case of annihilating heavy quarkonia, produced in a quark-gluon plasma (QGP) in a heavy ion collision at, e.g., the Large Hadron Collider, it is well known that their dissociation via the absorption of an on-shell gluon is not the dominant process for temperature larger than the binding energy (see, e.g., Refs. [62,63]). Instead, it is the dissociation via light quark and gluon scattering that dominates. Because of detailed balance, this implies that the dominant heavy quarkonia BSF channel must be via 
light parton scattering as well. Since the system of heavy quarks inside a QGP is similar to DM in the early Universe (where the latter contains many more relativistic species), this insight may have profound implications for DM models where light or massless mediators in coannihilation scenarios are involved.

In this work, we investigate a mediator whose mass is of the order of the $Z, W^{ \pm}$, or Higgs boson masses. This is of particular interest, since the binding and kinetic energies of WIMPs during their freeze-out could be insufficient to emit an on-shell electroweak-scale boson in order to form a bound state. In such a case, BSF has only marginal effects. However, inside a relativistic plasma background, scattering on bath particles via exchange of a virtual mediator can precipitate BSF. Such a process has no kinematic block and may entirely dominate over the on-shell mediator emission. Moreover, our work provides insight for models with very light or massless mediators coupled to a relativistic plasma, such as very heavy WIMPs decoupling before the electroweak phase transition, or scenarios of DM coannihilation with colored partners.

As a proof of concept, this article investigates for the first time BSF via scattering, in a simplified DM model that aims to resemble more realistic cases with $Z, W^{ \pm}, H$, or other exotic interactions. The details of the model and the computation based on a generalized BSF cross section [64], capturing higher-order processes in a proper thermal field theory framework, are shared in the section Model and cross sections. The numerical evaluation of the thermally averaged quantities and the implications of strongly enhanced BSF rates are discussed in the section Numerical results and implications. The final section is the section Conclusion.

Model and cross sections.-We consider a simple fourparameter model:

$$
\mathcal{L} \supset-g \bar{\chi} \gamma^{\mu} \chi V_{\mu}-g \sum_{i=1}^{N} \bar{b}_{i} \gamma^{\mu} b_{i} V_{\mu},
$$

where nonrelativistic DM $(\chi)$ with mass $m_{\chi}$ and the $N$ ultrarelativistic primordial plasma particles $(b)$ are Dirac fermions. The mass of the Abelian vector mediator $V^{\mu}$ is assumed to fulfill $m_{V} \lesssim \alpha m_{\chi} / 2$, where $\alpha \equiv g^{2} /(4 \pi)$ is the dark fine structure constant, such that bound state solutions exist in the DM particle-antiparticle spectrum.

The generalized BSF cross section, which includes the on-shell emission and the bath-particle scattering in Fig. 1, as well as other higher order processes in a proper thermal field theoretical framework, is given by [64] (see also Ref. [65])

$\sigma_{n l m}^{\mathrm{BSF}} v_{\text {rel }}=\int \frac{d^{3} p}{(2 \pi)^{3}} D_{\mu \nu}^{-+}(P) \sum_{\text {spins }} \mathcal{T}_{\mathbf{k}, n l m}^{\mu}(P) \mathcal{T}_{\mathbf{k}, n l m}^{\nu \star}(P)$.

$P=(\Delta E, \mathbf{p})$ is the $V^{\mu}$ four-momentum, whose energy component, $\Delta E \simeq \mathcal{E}_{\mathbf{k}}-\mathcal{E}_{n l m}>0$ [68], is the energy
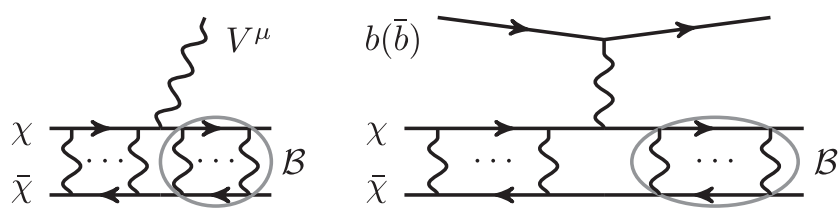

FIG. 1. Feynman diagrams for bound state formation via onshell mediator emission (left) and via bath-particle scattering (right) shown. Both processes contain also the diagram where the mediator is attached to $\bar{\chi}$.

dissipated in the inelastic conversion. It includes the kinetic energy of the relative motion, $\mathcal{E}_{\mathbf{k}}=\mathbf{k}^{2} /(2 \mu)=\mu v_{\text {rel }}^{2} / 2$, with $\mu=m_{\chi} / 2$ being the $\chi \bar{\chi}$ reduced mass, plus the absolute value of the binding energy $\mathcal{E}_{n l m}<0$ of the bound state with quantum numbers $n l m$. The Eq. (2) contains the closed-time-path two-point correlation function $D_{\mu \nu}^{-+}$of the vector boson, which is contracted with nonrelativistic (NR) scattering-to-bound transition matrix elements at the Born level. The integral over $\mathbf{p}$ picks out the various physical poles of $D_{\mu \nu}^{-+}$.

The matrix elements in the dipole approximation are

$$
\left.\mathcal{T}_{\mathbf{k}, n l m}^{\mu}(P) \equiv\left(g_{\chi} g_{\bar{\chi}} 4 m_{\chi}^{2} 2 M\right)^{-1 / 2} \mathcal{M}_{\mathbf{k}, n l m}^{\mu}\right|_{\mathrm{dip}} ^{\mathrm{NR}},
$$

where $M$ is the bound state mass and the initial DM spin d.o.f. are $g_{\chi} g_{\bar{\chi}}=4$. $\mathcal{M}^{\mu}$ is defined as the Fourier transform of the Scattering $(\mathcal{S})$-Bound $(\mathcal{B})$ state transition matrix element of the current operator, here given by

$$
\not^{4} \mathcal{M}_{\mathbf{k}, n l m}^{\mu}=\int d^{4} x e^{i P x}\left\langle\mathcal{B}_{n l m}\left|g \bar{\chi}(x) \gamma^{\mu} \chi(x)\right| \mathcal{S}_{\mathbf{k}}\right\rangle
$$

$\phi^{4}=(2 \pi)^{4} \delta^{4}$ is the four-momentum conserving delta function and spin indices are implicit.

In the following, we consider only the dominant capture into the ground state. Taking the nonrelativistic and dipole limits, we obtain [66]

$\sum_{\text {spins }} \mathcal{T}_{\mathbf{k}, 100}^{\mu} \mathcal{T}_{\mathbf{k}, 100}^{\nu \star}=\frac{4 \pi h(\zeta, \xi)}{\mu^{3} \Delta E^{2}}\left(\begin{array}{c}\mathbf{p} \cdot \hat{\mathbf{k}} \\ \Delta E \hat{\mathbf{k}}\end{array}\right)^{\mu}\left(\begin{array}{c}\mathbf{p} \cdot \hat{\mathbf{k}} \\ \Delta E \hat{\mathbf{k}}\end{array}\right)^{\nu}$,

with $\Delta E=\mathcal{E}_{\mathbf{k}}+\left|\mathcal{E}_{100}\right|$ for the ground state, and $\zeta \equiv \alpha / v_{\text {rel }}$, $\xi \equiv \alpha m_{\chi} /\left(2 m_{V}\right)$. As a check, $\mathcal{T}$ fulfills the current conservation $P_{\mu} T_{\mathbf{k}, 100}^{\mu}=0$ as a consequence of the global symmetry. Dimensionless $h(\zeta, \xi)$ contains the dipole approximation of the overlap integral, which can be computed only numerically for a massive mediator [66]. Note that once the Born transition matrix element of Eq. (4) is determined [e.g., Eq. (5)] it can be reused in Eq. (2) at any order of the perturbative expansion of the two-point correlation function.

The two-point correlation function in coordinate space $D_{\mu \nu}^{-+}(x, y) \equiv\left\langle V_{\mu}(x) V_{\nu}(y)\right\rangle$, where $\langle\ldots\rangle \propto \operatorname{Tr}\left[e^{-H_{\text {env }} / T} \ldots\right]$, encodes all interactions with the primordial plasma and 
hence the information of the bath particles. It is related to the spectral function $D^{\rho}$ by the Kubo-Martin-Schwinger relation (see, e.g., Sec. II in Ref. [69]):

$$
D_{\mu \nu}^{-+}(\Delta E, \mathbf{p})=\left[1+f_{V}^{\mathrm{eq}}(\Delta E)\right] D_{\mu \nu}^{\rho}(\Delta E, \mathbf{p}) .
$$

The equilibrium phase-space distribution $f_{V}^{\mathrm{eq}}$ obeys BoseEinstein statistics. It is convenient to compute the spectral function from the retarded correlator via $D_{\mu \nu}^{\rho}=2 \Im\left[i D_{\mu \nu}^{R}\right]$, since the latter obeys the Dyson-Schwinger equation, also in thermal field theory,

$$
D_{\mu \nu}^{R}=D_{\mu \nu}^{R, 0}+D_{\mu \alpha}^{R, 0} \Pi_{R}^{\alpha \beta} D_{\beta \nu}^{R, 0}+\cdots
$$

In the following, we show that BSF via on-shell mediator emission is reproduced from the free term, while BSF via bath-particle scattering is contained in the interaction term with the retarded self-energy $\Pi_{R}$. For both processes, Eq. (5) can be reused to calculate the BSF cross section Eq. (2), thanks to the factorization.

Although a self-energy with fermions is analyzed in this work, the formalism can be adapted to non-Abelian interactions, e.g., gluon scattering (triple vertex). For nonAbelian theories, Ref. [35] provides many expressions of the transition element of Eq. (3), ready to be used with Eq. (2). For scalar mediators, one can drop " $\mu \nu$ " everywhere and adjust Eq. (4) to Yukawa interactions according to Refs. [48,66,68,70-73].

Capture via on-shell mediator emission: To lowest order in perturbation theory the retarded propagator of the massive vector mediator is $D_{\mu \nu}^{R, 0}(P)=-i g_{\mu \nu}\left[P^{2}-m_{V}^{2}+\right.$ $\left.i \operatorname{sgn}\left(P^{0}\right) \epsilon\right]^{-1}$. Taking $2 \Im[i \ldots]$ of the latter to compute the spectral function, and inserting Eqs. (5) and (6) into the cross section, the known result for capture into the ground state via the on-shell emission of a massive vector mediator (mBSF) is reproduced after performing elementary integrals [cf. Eq. (3.7a) in Ref. [66] ]:

$\sigma_{100}^{\mathrm{mBSF}} v_{\text {rel }}=\frac{4 h(\zeta, \xi) \Delta E}{3 \mu^{3}}\left[1+f_{V}^{\mathrm{eq}}(\Delta E)\right] \frac{s_{\mathrm{ps}}^{1 / 2}\left(3-s_{\mathrm{ps}}\right)}{2}$,

where $\quad s_{\mathrm{ps}} \equiv 1-m_{V}^{2} / \Delta E^{2} \quad$ yields the kinematic suppression.

Capture via bath-particle scattering: The vector boson retarded self-energy generated by ultrarelativistic fermions is

$$
\begin{aligned}
\Pi_{\mu \nu}^{R}(\Delta E, \mathbf{p})= & g^{2} N \int d \Pi_{1} d \Pi_{2} \operatorname{Tr}\left[\gamma_{\mu} \not P_{1} \gamma_{\nu} P_{2}\right] \\
& \times\left\{\frac{i 2 \not \not^{3}\left(\mathbf{p}+\mathbf{p}_{1}-\mathbf{p}_{2}\right)}{\Delta E+\left|\mathbf{p}_{1}\right|-\left|\mathbf{p}_{2}\right|+i \epsilon}\right. \\
& \left.\times\left[f_{b}^{\mathrm{eq}}\left(\left|\mathbf{p}_{1}\right|\right)-f_{b}^{\mathrm{eq}}\left(\left|\mathbf{p}_{2}\right|\right)\right]+\cdots\right\} .
\end{aligned}
$$

The on-shell integration over the ultrarelativistic momenta is $d \Pi_{i}=\left[d^{3} p_{i} /(2 \pi)^{3} 2\left|\mathbf{p}_{i}\right|\right]$, and $P_{i}=\left(\left|\mathbf{p}_{i}\right|, \mathbf{p}_{i}\right)$. The second line contains Fermi-Dirac distributions. The term shown corresponds to bath-particle scattering. The omitted term contains an off-shell decay of the mediator into a bath particle-antiparticle pair, which we find to be subdominant compared to the scattering case. By taking the imaginary part of the propagator in the second line to compute the spectral function, and together with the transition elements in Eq. (5), we have for the first time computed the BSF cross section via bath-particle scattering (bBSF) for a massive vector mediator:

$$
\int \frac{d \Omega_{\mathbf{k}}}{4 \pi}\left(\sigma_{100}^{\mathrm{bBSF}} v_{\text {rel }}\right)=\frac{4 h(\zeta, \xi) \Delta E}{3 \mu^{3}} \times N \times R_{100}^{\mathrm{bBSF}} .
$$

The angular average arises in the thermal average of the cross section. We find it here for computational reasons meaningful to already perform. The first term is identical to the one appearing in Eq. (8) for the mBSF. $R_{100}^{\mathrm{bBSF}}$ encompasses the details of the bath-particle scattering and is given in dimensionless coordinates $y \equiv\left|\mathbf{p}_{1}\right| / T$ by

$$
\begin{aligned}
R_{100}^{\mathrm{bBS}}= & \frac{\alpha}{\pi}\left(\frac{T}{\Delta E}\right)^{3} \int_{0}^{\infty} d y \frac{1}{e^{y}+1}\left(1-\frac{1}{e^{\Delta E / T+y}+1}\right) \\
& \times\left\{\left[y^{2}+\left(\frac{\Delta E}{T}+y\right)^{2}+\frac{m_{V}^{2}}{T^{2}}\right] \ln \left[1+\frac{4 y(\Delta E / T+y)}{m_{V}^{2} / T^{2}}\right]\right. \\
& \left.-\frac{4 y(\Delta E / T+y)\left[m_{V}^{2} / T^{2}+(\Delta E / T+2 y)^{2}\right]}{m_{V}^{2} / T^{2}+4 y(\Delta E / T+y)}\right\}
\end{aligned}
$$

The amount of integrals were reduced analytically down to only a single one remaining, without further approximations. Both particle and antiparticle scattering are contained in Eq. (9). We stress that the bBSF has no kinematic block, and has an additional temperature and velocity-enhancement factor $(T / \Delta E)^{3}$ for $T \gg\left|\mathcal{E}_{100}\right|$. We explicitly checked that Eq. (10) is identical to the expression obtained in the Boltzmann formalism. The benefit of the proper thermal field theory definition of Eq. (2) in comparison to the Boltzmann formalism is that the interference terms arising from the imaginary part of the double mediator propagator in Eq. (7), are automatically taken into account. In the Boltzmann framework they are absent. While for $m_{V} \gg$ $\left|\mathcal{E}_{100}\right|$ these interference terms are negligible, they regulate Eq. (11) in the limit $m_{V} \rightarrow 0$, by canceling the forward scattering divergence of the bath particles, as proven in Ref. [64]. In the latter work, it is also shown that the UVdivergent vacuum parts in Eq. (9) do not contribute significantly after standard renormalization. This shows the power of Eq. (2). It contains all possible processes up to the chosen order in the coupling expansion.

Numerical results and implications.-The mBSF and bBSF thermal averages, Eqs. (8) and (10), are compared in Fig. 2. Two cases with $m_{V} \gtrsim\left|\mathcal{E}_{100}\right|$ are shown. In the left 

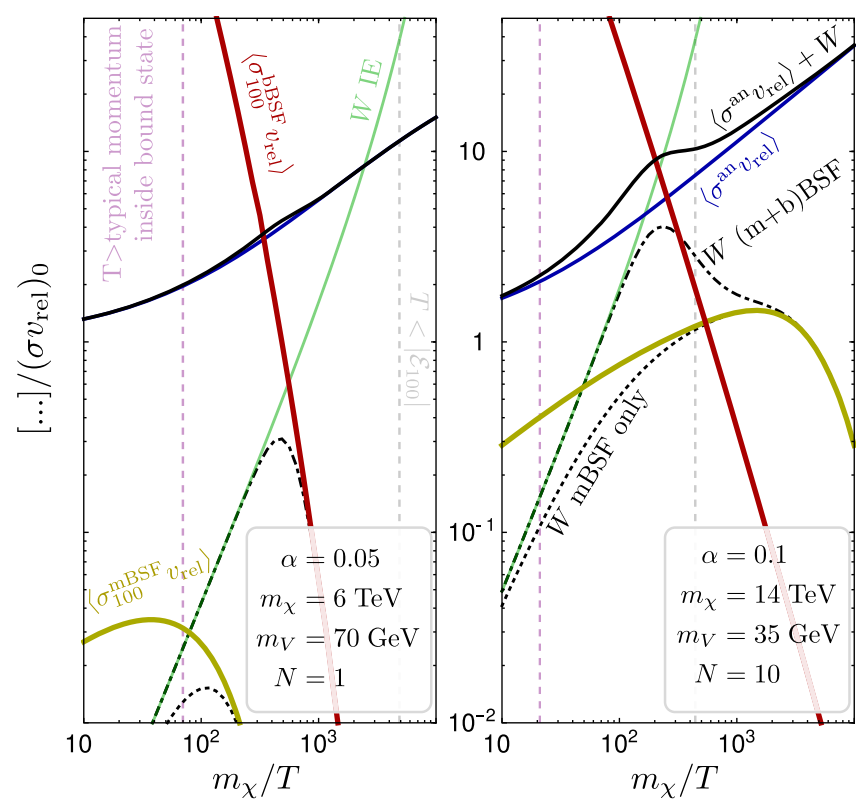

FIG. 2. Thermally averaged velocity-weighted cross sections $\left\langle\sigma v_{\text {rel }}\right\rangle$ for ground-state capture via bath-particle scattering (red) and on-shell mediator emission (yellow). Everything is normalized to the tree-level value for annihilation, $\left(\sigma v_{\text {rel }}\right)_{0} \equiv$ $(1+N) \pi \alpha^{2} / m_{\chi}^{2}$, where $N$ is the bath fermion species. The sum of the SE annihilation (blue) and the effective BSF cross section $W$ determines the DM depletion (black solid). The vertical grey dashed lines mark the temperatures equal to the binding energies, $\left|\mathcal{E}_{100}\right| \simeq 1.23 \mathrm{GeV}$ and $31.6 \mathrm{GeV}$. Including the bBSF reduces the DM density by about $1.5 \%$ and $20 \%$. While the DM density departs from equilibrium at $m_{\chi} / T \approx 25-30$, at that time it is still more than an order of magnitude larger than its final value. The latter is reached within $5 \%$ at $m_{\chi} / T \approx 2600$ and 4400 .

panel, only the ground state close to the threshold exists, aiming to parametrically resemble Wino-like DM scenarios. In the right panel, the coupling is similar to the strong coupling of the SM. The ground state is nearly Coulombic, aiming to provide insight into, e.g., coannihilation with colored particles (neutralino-squark), or multi-TeV WIMPs, typically residing in higher $S U(2)_{L}$ representations. The results prove the concept: the bBSF can dominate over the mBSF.

The reasons are qualitatively the following. First, as seen in Eq. (10), the bBSF is enhanced at high temperatures by the large number density of the ultrarelativistic bath particles, $n_{b} \propto T^{3}$. Second, while the mBSF is suppressed by the smallness of the dissipated energy $\left(\sigma^{\mathrm{mBSF}} v_{\text {rel }}\right) \propto$ $|\mathbf{p}|=\Delta E$ [66], the bBSF is enhanced by it: $\left(\sigma^{\mathrm{bBSF}} v_{\text {rel }}\right) \propto$ $1 / \Delta E^{2}$ has a stronger dependence on the inverse relative velocity. This is partly because the momentum of the offshell $V^{\mu}$ is not fixed by $\Delta E$, as is in the mBSF. These features yield the relative scaling $R^{\mathrm{bBSF}} \propto(T / \Delta E)^{3}$. Finally, in sharp contrast to the mBSF, the bBSF is kinematically accessible even if $m_{V} \gtrsim \Delta E$. An important similarity, however, is that both processes follow the same selection rules, seen from the factorization in Eq. (2). Hence, forbidden channels can also not be accessed via higher order processes contained in the mediator spectral function.

The implications of a large bBSF rate on the evolution of the DM density can be seen from the Boltzmann equation including the lowest bound states [28,29],

$$
\begin{aligned}
& \dot{n}_{\chi}+3 H n_{\chi}=-\left[\left\langle\sigma^{\mathrm{an}} v_{\mathrm{rel}}\right\rangle+W(T)\right]\left[n_{\chi}^{2}-\left(n_{\chi}^{\mathrm{eq}}\right)^{2}\right], \\
& W \equiv\left\langle\sigma_{100}^{\mathrm{BSF}} v_{\mathrm{rel}}\right\rangle\left[\frac{(1 / 4) \Gamma_{100, S}^{\mathrm{dec}}}{\Gamma_{100, S}^{\mathrm{dec}}+\Gamma_{100}^{\mathrm{dis}}}+\frac{(3 / 4) \Gamma_{100, T}^{\mathrm{dec}}}{\Gamma_{100, T}^{\mathrm{dec}}+\Gamma_{100}^{\mathrm{dis}}}\right] .
\end{aligned}
$$

The DM depletion depends on the Sommerfeld enhanced annihilation cross section and the effective BSF cross section $W$. The latter consists of the total thermally averaged BSF cross section, weighted by the branching fractions of the spin-singlet and triplet bound state decay into $V V$ and $b \bar{b}$ pairs, respectively. The factors $1 / 4$ and $3 / 4$ account for the spin multiplicities. The dissociation rate is related via detailed balance to the BSF, $\Gamma_{100}^{\mathrm{dis}}=$ $\left\langle\sigma_{100}^{\mathrm{BSF}} v_{\text {rel }}\right\rangle\left(n_{\chi}^{\mathrm{eq}}\right)^{2} / n_{100}^{\mathrm{eq}}$, where $n_{100}^{\mathrm{eq}}$ is the ground state equilibrium number density with four spin d.o.f.

The effective cross section has two asymptotic regimes:

$$
W(T) \simeq \begin{cases}\Gamma_{100}^{\mathrm{dec}} n_{100}^{\mathrm{eq}} /\left(n_{\chi}^{\mathrm{eq}}\right)^{2} & \text { for } \Gamma_{100, i}^{\mathrm{dec}} \ll \Gamma_{100}^{\mathrm{dis}}, \\ \left\langle\sigma_{100}^{\mathrm{BSF}} v_{\mathrm{rel}}\right\rangle & \text { for } \Gamma_{100, i}^{\mathrm{dec}} \gg \Gamma_{100}^{\mathrm{dis}},\end{cases}
$$

where $\Gamma_{100}^{\mathrm{dec}}=\left(\Gamma_{100, S}^{\mathrm{dec}}+3 \Gamma_{100, T}^{\mathrm{dec}}\right) / 4$. In the first regime, the system is in Saha ionization equilibrium (IE), where $W$ is (i) independent of the BSF cross section [69], and (ii) maximal for a given temperature and bound state decay rate. The maximum size of $W$ is shown by the green line in Fig. 2, and grows exponentially for $T \lesssim\left|\mathcal{E}_{100}\right|$. W starts to deviate from its IE value when $\Gamma_{100, i}^{\mathrm{dec}} \gtrsim \Gamma_{100}^{\mathrm{dis}}$. Importantly, this transition depends on the total BSF cross section contained in $\Gamma_{100}^{\mathrm{dis}}$. In this second asymptotic regime, attained at later times, $W$ is the actual BSF cross section, since the bound states immediately decay without being dissociated into scattering states. The above imply that the bBSF increases the DM depletion both because it sustains IE until lower temperatures than the mBSF alone, and because it may enhance or dominate the total BSF rate after the end of IE, even if temporarily so, as seen in Fig. 2.

Overall, the bBSF effect on the DM density depends on (a) the difference between $W$ with and without the bBSF, and the interval within which this is significant, and (b) the magnitude of $W$ relatively to annihilation.

Figure 2 clearly demonstrates that (a) can be very significant. On the left panel, this is largely due to the mBSF being kinematically suppressed, while on the right panel it is in part due to the large $N$; while here we used $N=10$, an even larger number of relativistic d.o.f. couple to the SM force mediators during the decoupling of 
TeV-scale DM. Note that because the cross sections increase as $T$ drops, the DM depletion remains significant even after the traditional freeze-out at $m_{\chi} / T \approx 30$. Importantly, this may encompass a period after electroweak symmetry breaking, when $\mathrm{mBSF}$ via $W^{ \pm}, Z$ emission is kinematically suppressed, even if $m_{\chi} \gtrsim 5 \mathrm{TeV}$, for which freeze-out begins in the symmetric phase.

The right panel of Fig. 2 shows also that (b) can be significant. While in the present model $\left\langle\sigma^{\text {an }} v_{\text {rel }}\right\rangle$ scales with $N$, thus tempering the relative importance of the bBSF, this is not generally the case. For example, a gauge-singlet scattering state in non-Abelian theories cannot annihilate via an off-shell $s$-channel gauge boson into bath particles (see, e.g., Ref. [35]); then the bBSF may potentially have a larger relative effect. On the left panel, $W$ enhances the DM depletion very minimally. However, the phenomenological importance of the bBSF depends also on the sensitivity of the experimental probes. Here, even a few percent difference can have dramatic implications. Indeed, the most powerful probe of $\mathrm{TeV}$-scale $\mathrm{DM}$ is indirect detection, and the indirect signals of $\mathrm{TeV}$-scale WIMPs exhibit sharp parametric resonances that render the viability of a model very sensitive to the predicted DM mass.

The dipole approximation for the bBSF breaks down at $T$ larger than the typical momentum inside the bound state, marked by the pink dashed line in Fig. 2. However, since the system is robustly in IE, the precise BSF cross section is irrelevant. Nevertheless, the thermal bath at high $T$ may affect the SE annihilation cross section and the bound state decay rate that determine the collision term in Eq. (12) in IE.

The impact of the primordial plasma on the SE annihilation and bound state decay rate has already been studied within a different thermal field theory approach $[33,36,37,39,41,69,74-77]$. In particular, Eq. (7) can be resummed in the hard-thermal-loop approximation [78], giving an effective in-medium potential for a Schrödingerlike equation, including real part corrections (e.g., Debye screening), as well as an imaginary thermal width $[79,80]$. A most conservative estimate [74] shows that the latter leads to melting of the bound states (see, e.g., Fig. 1 in Ref. [81] for data) for $T$ to the left of the pink dashed line, where ultraefficient bath-particle scattering strongly mixes the bound and scattering states.

The formal key point of this work is that the validity region of the generalized BSF cross section in Eq. (2) [64] has overlap with the formalism used in Refs. [33,36,37,39,41,69,74-77], where the latter is strictly speaking limited to IE $[69,77]$. Combined, we have achieved a complete procedure to accurately calculate thermal relics in the early Universe for any temperature regime, ranging from the melting of bound states at high $T$ down to far below their decoupling from ionization equilibrium.

Conclusion.-The early Universe contained more than 100 relativistic d.o.f. that may have contributed to DM bound state formation via scattering. In this work, we analyzed the contribution of relativistic fermions coupled to DM through a massive vector mediator. While the BSF via on-shell emission of a mediator may be suppressed in such a case, we have demonstrated that capture via scattering on bath particles can be highly active. In addition, we showed that the latter can keep the system in ionization equilibrium for longer time, maximizing the depletion rate at a given temperature. Our results imply that bound state effects during WIMP thermal decoupling may be more severe than previously estimated, consequently allowing for DM to be heavier than expected. Future detailed calculations of these effects will inform our direct, indirect, and collider searches.

T. B. was supported by World Premier International Research Center Initiative (WPI), MEXT, Japan. K. P. was supported by the ANR ACHN grant ("TheIntricateDark" project), and by the NWO Vidi grant "Self-interacting asymmetric dark matter." We are thankful to Burkhard Blobel, Peter Cox, Julia Harz and Shigeki Matsumoto for very stimulating discussions, as well as to Satoshi Shirai for checking the manuscript.

*tobias.binder@ipmu.jp

†kyohei.mukaida@desy.de

${ }^{*}$ kpetraki@lpthe.jussieu.fr

[1] B. W. Lee and S. Weinberg, Phys. Rev. Lett. 39, 165 (1977).

[2] J. R. Ellis, J. S. Hagelin, D. V. Nanopoulos, K. A. Olive, and M. Srednicki, Nucl. Phys. B238, 453 (1984).

[3] G. Arcadi, M. Dutra, P. Ghosh, M. Lindner, Y. Mambrini, M. Pierre, S. Profumo, and F. S. Queiroz, Eur. Phys. J. C 78, 203 (2018).

[4] P. A. R. Ade et al. (Planck Collaboration), Astron. Astrophys. 594, A13 (2016).

[5] J. Hisano, S. Matsumoto, M. Nagai, O. Saito, and M. Senami, Phys. Lett. B 646, 34 (2007).

[6] A. Sommerfeld, Ann. Phys. (Berlin) 403, 257 (1931).

[7] A. D. Sakharov, Zh. Eksp. Teor. Fiz. 18, 631 (1948) [Usp. Fiz. Nauk 161, 29 (1991)].

[8] K. Belotsky, D. Fargion, M. Khlopov, and R. V. Konoplich, Phys. At. Nucl. 71, 147 (2008).

[9] A. Freitas, Phys. Lett. B 652, 280 (2007).

[10] M. Cirelli, A. Strumia, and M. Tamburini, Nucl. Phys. B787, 152 (2007).

[11] C. F. Berger, L. Covi, S. Kraml, and F. Palorini, J. Cosmol. Astropart. Phys. 10 (2008) 005.

[12] A. Hryczuk, R. Iengo, and P. Ullio, J. High Energy Phys. 03 (2011) 069.

[13] A. Hryczuk, Phys. Lett. B 699, 271 (2011).

[14] K. Harigaya, K. Kaneta, and S. Matsumoto, Phys. Rev. D 89, 115021 (2014).

[15] S. El Hedri, A. Kaminska, and M. de Vries, Eur. Phys. J. C 77, 622 (2017).

[16] M. Beneke, A. Bharucha, A. Hryczuk, S. Recksiegel, and P. Ruiz-Femenia, J. High Energy Phys. 01 (2017) 002. 
[17] M. Beneke, A. Bharucha, F. Dighera, C. Hellmann, A. Hryczuk, S. Recksiegel, and P. Ruiz-Femenia, J. High Energy Phys. 03 (2016) 119.

[18] T. R. Slatyer, J. Cosmol. Astropart. Phys. 02 (2010) 028.

[19] M. Beneke, C. Hellmann, and P. Ruiz-Femenia, J. High Energy Phys. 05 (2015) 115.

[20] K. Blum, R. Sato, and T. R. Slatyer, J. Cosmol. Astropart. Phys. 06 (2016) 021.

[21] E. Braaten, E. Johnson, and H. Zhang, J. High Energy Phys. 11 (2017) 108.

[22] E. Braaten, E. Johnson, and H. Zhang, J. High Energy Phys. 05 (2018) 062.

[23] E. Braaten, E. Johnson, and H. Zhang, J. High Energy Phys. 02 (2018) 150.

[24] Y.-L. Tang and G.-L. Zhou, Phys. Rev. D 99, 036016 (2019).

[25] M. Pospelov and A. Ritz, Phys. Lett. B 671, 391 (2009).

[26] J. D. March-Russell and S. M. West, Phys. Lett. B 676, 133 (2009).

[27] W. Shepherd, T. M. P. Tait, and G. Zaharijas, Phys. Rev. D 79, 055022 (2009).

[28] B. von Harling and K. Petraki, J. Cosmol. Astropart. Phys. 12 (2014) 033.

[29] J. Ellis, J. L. Evans, F. Luo, and K. A. Olive, J. High Energy Phys. 02 (2016) 071.

[30] S. P. Liew and F. Luo, J. High Energy Phys. 02 (2017) 091.

[31] P. Asadi, M. Baumgart, P. J. Fitzpatrick, E. Krupczak, and T. R. Slatyer, J. Cosmol. Astropart. Phys. 02 (2017) 005 [arXiv:1611.06212].

[32] E. Johnson, E. Braaten, and H. Zhang, Proc. Sci. ICHEP2016 (2016) 210.

[33] S. Kim and M. Laine, J. High Energy Phys. 07 (2016) 143.

[34] A. Mitridate, M. Redi, J. Smirnov, and A. Strumia, J. Cosmol. Astropart. Phys. 05 (2017) 006.

[35] J. Harz and K. Petraki, J. High Energy Phys. 07 (2018) 096.

[36] S. Biondini and M. Laine, J. High Energy Phys. 04 (2018) 072.

[37] S. Biondini and S. Vogl, J. High Energy Phys. 02 (2019) 016.

[38] H. Fukuda, F. Luo, and S. Shirai, J. High Energy Phys. 04 (2019) 107.

[39] S. Biondini and S. Vogl, J. High Energy Phys. 11 (2019) 147.

[40] J. Harz and K. Petraki, Phys. Rev. D 97, 075041 (2018).

[41] S. Biondini, J. High Energy Phys. 06 (2018) 104.

[42] J. Harz and K. Petraki, J. High Energy Phys. 04 (2019) 130.

[43] A. Kamada, M. Kaplinghat, A. B. Pace, and H.-B. Yu, Phys. Rev. Lett. 119, 111102 (2017).

[44] M. Kaplinghat, T. Ren, and H.-B. Yu, arXiv:1911.00544.

[45] M. R. Buckley and P. J. Fox, Phys. Rev. D 81, 083522 (2010).

[46] L. G. van den Aarssen, T. Bringmann, and C. Pfrommer, Phys. Rev. Lett. 109, 231301 (2012).

[47] S. Tulin, H.-B. Yu, and K. M. Zurek, Phys. Rev. D 87, 115007 (2013).

[48] L. Pearce and A. Kusenko, Phys. Rev. D 87, 123531 (2013).

[49] K. Petraki, L. Pearce, and A. Kusenko, J. Cosmol. Astropart. Phys. 07 (2014) 039.

[50] L. Pearce, K. Petraki, and A. Kusenko, Phys. Rev. D 91, 083532 (2015).
[51] T. Bringmann, F. Kahlhoefer, K. Schmidt-Hoberg, and P. Walia, Phys. Rev. Lett. 118, 141802 (2017).

[52] H. An, M. B. Wise, and Y. Zhang, Phys. Rev. D 93, 115020 (2016).

[53] M. Cirelli, P. Panci, K. Petraki, F. Sala, and M. Taoso, J. Cosmol. Astropart. Phys. 05 (2017) 036.

[54] I. Baldes and K. Petraki, J. Cosmol. Astropart. Phys. 09 (2017) 028.

[55] I. Baldes, M. Cirelli, P. Panci, K. Petraki, F. Sala, and M. Taoso, Sci. Post Phys. 4, 041 (2018).

[56] A. Kamada, K. Kaneta, K. Yanagi, and H.-B. Yu, J. High Energy Phys. 06 (2018) 117.

[57] A. Kamada, M. Yamada, and T. T. Yanagida, J. High Energy Phys. 03 (2019) 021.

[58] S. Matsumoto, Y.-L. S. Tsai, and P.-Y. Tseng, J. High Energy Phys. 07 (2019) 050.

[59] A. Kamada, M. Yamada, and T. T. Yanagida, arXiv: 1908.00207.

[60] P. Ko, T. Matsui, and Y.-L. Tang, arXiv:1910.04311.

[61] M. Geller, S. Iwamoto, G. Lee, Y. Shadmi, and O. Telem, J. High Energy Phys. 06 (2018) 135.

[62] N. Brambilla, M. A. Escobedo, J. Ghiglieri, and A. Vairo, J. High Energy Phys. 05 (2013) 130.

[63] J. Hong and S. H. Lee, Phys. Rev. C 99, 034905 (2019).

[64] T. Binder, B. Blobel, J. Harz, and K. Mukaida, arXiv: 2002.07145.

[65] See Supplemental Material at http://link.aps.org/supplemental/ 10.1103/PhysRevLett.124.161102 for an alternative derivation of the cross section and conventions, which includes Refs. [64,66,67].

[66] K. Petraki, M. Postma, and J. de Vries, J. High Energy Phys. 04 (2017) 077.

[67] M. Beneke, F. Dighera, and A. Hryczuk, J. High Energy Phys. 10 (2014) 045; 07 (2016) 106(E).

[68] K. Petraki, M. Postma, and M. Wiechers, J. High Energy Phys. 06 (2015) 128.

[69] T. Binder, L. Covi, and K. Mukaida, Phys. Rev. D 98, 115023 (2018).

[70] M. B. Wise and Y. Zhang, Phys. Rev. D 90, 055030 (2014).

[71] H. An, M. B. Wise, and Y. Zhang, Phys. Lett. B 773, 121 (2017).

[72] R. Oncala and K. Petraki, J. High Energy Phys. 01 (2019) 070.

[73] R. Oncala and K. Petraki, J. High Energy Phys. 02 (2020) 036.

[74] S. Kim and M. Laine, J. Cosmol. Astropart. Phys. 01 (2017) 013.

[75] S. Biondini and M. Laine, J. High Energy Phys. 08 (2017) 047.

[76] S. Kim and M. Laine, Phys. Lett. B 795, 469 (2019).

[77] S. Biondini, S. Kim, and M. Laine, J. Cosmol. Astropart. Phys. 10 (2019) 078.

[78] E. Braaten and R. D. Pisarski, Nucl. Phys. B337, 569 (1990).

[79] M. Laine, O. Philipsen, P. Romatschke, and M. Tassler, J. High Energy Phys. 03 (2007) 054.

[80] N. Brambilla, J. Ghiglieri, A. Vairo, and P. Petreczky, Phys. Rev. D 78, 014017 (2008).

[81] A. M. Sirunyan et al. (CMS Collaboration), Phys. Lett. B 790, 270 (2019). 